\title{
Aproximación Cuantitativa para Valorar los Requisitos Curriculares en un Plan de Estudios
}

\author{
J. Juan D. Martínez Arboleda, Mabel Torres-Taborda, Hader Alzate Gil y Carlos Ocampo-López \\ Facultad de Ingeniería Química, Universidad Pontificia Bolivariana, Circular 1 No. 70 - 01, Medellín, \\ Colombia (e-mail: juandavid.martinez@upb.edu.co)
}

Recibido Jul. 31, 2015; Aceptado Oct. 1, 2015; Versión final Nov. 27, 2015, Publicado Abr. 2016

\begin{abstract}
Resumen
El presente trabajo propone una metodología para valorar el rigor de los requisitos de un currículo para un estudiante de educación superior, expresados en términos de requisitos y co-requisitos. Dicha metodología emplea un sistema de ponderación para las restricciones con base en la severidad y el impacto que estas restricciones representan en el flujo curricular, y define un índice de restricción. Ésta se puede aplicar desde un curso de un solo semestre hasta un plan de estudios completo. La metodología fue diseñada por la Facultad de Ingeniería Química de la Universidad Pontificia Bolivariana y aplicada al plan de estudios vigente en el 2014 y a un plan de estudios actualizado, donde el índice de restricción disminuyó de 0.54 a 0.38 . Así, el uso de un sistema de ponderación de las restricciones permite obtener una amplia perspectiva de su influencia en el plan de estudios y tomar decisiones hacia un currículo más flexible.
\end{abstract}

Palabras clave: currículo; requisitos; co-requisitos; índice de restricción; flujo curricular; flexibilidad

\section{Quantitative Approach to Evaluate Curriculum Requirements in a Curriculum}

\begin{abstract}
This work proposes a methodology to assess the rigor of curriculum requirements for a higher education student, expressed in terms of requisites and co-requisites. This methodology uses a weighting of restriction system based on the severity and impact that these restrictions represent on curriculum flow, and defines a constraint index. The method can be applied in different contexts, from a one-semester course to a full curriculum of a university career. The methodology was designed by the Chemical Engineering Department at the Universidad Pontificia Bolivariana in Colombia and it was applied to a curriculum of 2014 and to an updated curriculum. The constraint index decreased from 0.54 to 0.38 . Thus, the use of a weighting of restrictions system allows getting insights of their influence on curriculum in a wider way and helps in the decision making process towards a more flexible curriculum.
\end{abstract}




\section{INTRODUCCIÓN}

Las exigencias en la educación de los ingenieros, extendidas a nivel mundial como un efecto de la globalización; así como una disposición a mejorar el desempeño de los estudiantes y la preocupación ante la reducción del interés que tienen los jóvenes en seguir carreras de ingeniería, llamadas pregrados en varios países latinoamericanos como Colombia, han motivado que se hayan disparado las reformas curriculares, especialmente en la última década (Duque et al., 2011), tendencia a la que no ha sido ajena la Facultad de Ingeniería Química de la Universidad Pontificia Bolivariana (UPB). Según Soria y Mumpower (2012), los prerrequisitos fortalecen la capacidad de los estudiantes para la toma de decisiones, reducen el tiempo invertido en asesorías frente a errores cometidos y promueven la igualdad en el registro de cursos. En el ámbito académico, sólo dos razones justifican la imposición de un curso como prerrequisito: aumentar significativamente la probabilidad de éxito en el curso posterior o garantizar condiciones favorables para el bienestar de los estudiantes (Abou-Sayf, 2008).

El manejo de prerrequisitos es un asunto delicado para la comunidad académica. Por un lado, si los prerrequisitos son insuficientes conducen a una preparación profesional inadecuada, caracterizada por bajos rendimientos. Esto conlleva a su vez, a un aumento en las tasas de deserción de los programas y a una caída en las tasas de éxito. Por otro lado, un exceso de prerrequisitos conduce a una prolongación innecesaria del tiempo para graduarse y, en consecuencia, un aumento de la probabilidad de deserción (California Office of Administrative Law, 2008). En muchas instituciones, el número de requisitos previos se ha incrementado rápidamente en los últimos años, lo que lleva a la administración a invertir en un gran esfuerzo para examinar tanto los procesos utilizados para establecer los requisitos previos y la validez de los requisitos actuales. Por ejemplo, el Código de Regulaciones de California, enfatiza sobre la necesidad de justificar los requisitos previos a través de una cuidadosa revisión, donde se especifica que los prerrequisitos o correquisitos se establecerán para un curso cuando el comité de la universidad o el plan de estudios de distrito lo determinen. (California Office of Administrative Law, 2008). En California, por ejemplo, el código de regulaciones en educación especifica que los pre y correquisitos se establecen cuando el comité de currículo determina, basado en una revisión de los esquemas de registro académico, si es probable que un estudiante reciba una calificación poco satisfactoria a menos que éste tenga conocimientos o habilidades que no se enseñan en el curso. Con esta reflexión se podría controlar la proliferación de pre y correquisitos (California Office of Administrative Law, 2008).

Se utilizan varios enfoques para identificar pre y correquisitos. Un enfoque cualitativo, y en ocasiones subjetivo, es el empleado comúnmente por los docentes, el cual consiste en comparar las habilidades y competencias que debe poseer un estudiante frente a un curso objetivo, y definir un estatus de prerrequisito a aquellos cursos anteriores que aporten al desarrollo de dichas habilidades. Los enfoques cuantitativos se basan en técnicas estadísticas utilizadas para comparar el desempeño de los estudiantes que han completado el curso prerrequisito frente a aquellos que no lo hicieron. Rovick et al. (1999), Simpson y Eddy (1991) y Smith (1980) presentaron ejemplos de enfoques cualitativos utilizados para determinar los requisitos previos (Abou- Sayf, 2008). Además, Armstrong (1998); Cohen, Friedlander, Kelemen-Lohnas, \& Elmore (1990), Cramer \& Liberty (1981) y Wilson (1994) mencionan que los estudios basados en enfoques cuantitativos son frecuentes en la literatura revisada (Abou- Sayf, 2008).

En general, las investigaciones anteriores coinciden en que los pre y correquisitos son eficaces para mejorar el rendimiento estudiantil en los cursos de destino correspondientes (Woosley \& Callahan, 2011). Es por ello que reviste de gran importancia la planeación de la estructura de un currículo, garantizando la permanencia de los estudiantes y asegurando un flujo curricular controlado que le permita altas probabilidades de éxito en un programa (Baard \& Watts, 2008). La disposición de los pre y correquisitos en un plan de estudios, se podría relacionar con una de las formas de flexibilidad curricular: según Ruíz et al. (2007) esta flexibilidad se entiende como la posibilidad de que el estudiante construya su propio currículo. Desde el diseño de los planes de estudio esto implica la oferta de variantes a los estudiantes, lo que permite atender sus intereses y motivaciones, potenciar su autonomía y lograr una mayor satisfacción de las diferencias individuales.

Este tipo de flexibilidad puede cobijar el orden en el que se cursan las asignaturas en el plan de estudios, lo que exhibe ventajas tales como elevar los niveles de permanencia y de egreso de las carreras universitarias, y disminuir el número de bajas y de repitentes. De este modo, la flexibilidad puede estar presente como parte de las propuestas de políticas y estrategias que un sistema educacional se plantee, siempre que se garantice no afectar los niveles de calidad de la formación (Ruíz, et al., 2007). En vista de la importancia que tiene una correcta selección de los pre y correquisitos en el flujo curricular, es necesario una definición objetiva de los mismos basada en los contenidos de las asignaturas y las competencias que se requieren en los estudiantes. Una vez definidas estas necesidades, se ha de buscar racionalizar las restricciones para que un estudiante avance en su pensum sin que se afecte su formación como futuro profesional. Así, el conjunto de prerrequisitos en un plan de estudios se debe revisar para viabilizar la permanencia y el egreso de los estudiantes en el sistema (Zambrano y otros, 2005). 


\section{CONTEXTUALIZACIÓN}

Un plan de estudios tradicional o pensum corresponde al listado de actividades curriculares que se conectan entre sí y que se encuentran organizadas en una línea de tiempo secuencial. Una manera de hacer dicha conexión es mediante los pre y correquisitos, que se refieren a un conjunto de contenidos, habilidades o competencias que un estudiante debe haber cursado, aprobado o desarrollado para poder avanzar un siguiente paso en su plan de estudios. Un prerrequisito se refiere a los contenidos, habilidades o competencias que el estudiante debe cubrir o desarrollar, obteniendo o sobrepasando en su calificación definitiva un valor específico, como exigencia para que pueda matricular una determinada actividad curricular, o asignatura, de su interés. Por ejemplo, cuando un estudiante debe haber obtenido una calificación mayor o igual a 2.0 sobre 5.0 en la asignatura A, para poder matricular la asignatura B. O cuando un estudiante debe haber cursado y aprobado un cierto porcentaje del total de créditos del plan de estudios para matricular una actividad curricular específica, o como es común para la práctica industrial, donde es deseable que el estudiante que desee realizarla haya aprobado, por ejemplo, un $75 \%$ de su plan de estudios.

Según Soria y Mumpower (2012), se define como prerrequisito al medio por el cual un estudiante puede satisfacer los requerimientos de la institución para inscribirse en un curso. Los prerrequisitos podrían incluir la transferencia de crédito de otra institución, o una puntuación mínima en una prueba de nivel. Algunos restringen la inscripción del estudiante dentro de un sistema de registro informático, mientras que otros dependen de asesores académicos para registrar a los estudiantes en los cursos para inscribirlos en la secuencia apropiada de los mismos (University of Washington, 2015). Por otra parte, un correquisito se refiere a contenidos, habilidades o competencias con las que el estudiante debe estar apenas familiarizado para matricular otra actividad de su interés. Por ejemplo, se requiere que el estudiante haya cursado actividades curriculares que cubran o desarrollen esos contenidos o competencias, cualquiera sea la calificación definitiva que haya obtenido en ellas; o que el estudiante matricule la actividad curricular de su interés, y sus correquisitos, de manera simultánea. Es importante anotar que, si un estudiante cancela una actividad académica en curso, se le cancelan de manera automática las demás actividades que funcionen como sus correquisitos y que se encuentre asimismo cursando.

Llevando el tema a las facultades de Ingeniería, cuando el estudiante va a tomar un curso del plan de estudios, se debe analizar cuales cursos previos requiere haber visto, lo cual está determinado por los prerrequisitos asignados a dicho curso. En la mayoría de los casos, respecto a estos cursos previos exigidos, no se tiene en cuenta la nota final obtenida, sino que se haya visto o aprobado (Universidade de Sao Paulo, 2011; Universidad de Antioquia, 2015; Universidad de la Sabana, 2015). Para la Facultad de Ingeniería Química de la UPB, los pre y correquisitos pueden ser de tres clases: de contenidos, reguladores o formativos. Requisitos de contenidos se refieren a los que son estrictamente necesarios para que un estudiante se desempeñe bien en la actividad académica que los exige. Requisitos formativos son aquellos que, si bien no son estrictamente necesarios para que un estudiante se desempeñe bien en una determinada actividad académica, hay factores que hacen que sí sea deseable que ya los haya cubierto; por ejemplo, es deseable que para asumir algunos cursos de fin de carrera, el estudiante ya haya aprobado todas las ciencias básicas. Requisitos reguladores son exigencias que tienen en cuenta la madurez de un estudiante para abordar actividades curriculares complejas, como la práctica industrial o el trabajo de grado, madurez que es determinada por entrevistas con instancias como profesores asesores.

Existen dos razones básicas por las cuales el Comité de currículo de la Facultad de Ingeniería Química ha deseado valorar las restricciones de los pre y correquisitos dentro del plan de estudios: las múltiples solicitudes de los estudiantes al Consejo de Facultad para realizar excepciones y levantar temporalmente uno o más de los pre y correquisitos de una determinada actividad académica; y los fuertes efectos de la cancelación automática de los cursos que funcionan como correquisitos de alguno de ellos, que el estudiante se encuentre cursando y que decida cancelar por cualquier motivo. Por lo anterior, el Comité decidió realizar una revisión del conjunto de pre y correquisitos del plan de estudios vigente, para lo cual creó una herramienta de análisis para la valoración de las restricciones en el flujo curricular que permitiera tomar decisiones con elementos objetivos. El avance o flujo curricular se refiere al tránsito que realiza el estudiante por el plan de estudios de la carrera, a medida que va aprobando asignaturas para acceder a las siguientes (Pino Vera \& Soto Narbona, 2013).

El desarrollo de esta investigación ha demostrado que muchas veces se establecen normas sin tener bases sólidas que lo justifiquen. Eso es observable en que a menudo se imponen prerrequisitos para asignaturas bajo supuestos erróneos, los que pueden afectar de forma negativa a personas y procesos (Pino Vera \& Soto Narbona, 2013). 
La revisión, y la herramienta desarrollada, se realizaron en el marco de los lineamientos para la transformación curricular que se lleva a cabo en el programa de Ingeniería Química en cuanto a duración, créditos académicos y flexibilidad; también se tuvieron en cuenta las sugerencias recibidas de parte de pares académicos y del gobierno en el proceso de renovación de la Acreditación de Alta Calidad, renovación recibida en 2011; y, por último, se ajustaron a las intencionalidades del Modelo pedagógico de la UPB, donde se indica que la Universidad propende por currículos integrados, flexibles, contextualizados e interdisciplinarios (Universidad Pontificia Bolivariana, 2009). En última instancia, la revisión de las restricciones del currículo nace de la conciencia que la Facultad ha adquirido sobre la importancia de actualizar permanentemente el currículo de su pregrado y su conexión con la realidad cultural y laboral actual, a nivel nacional e internacional, como lo indican Castrillón y Garcés (2012).

Es pertinente aclarar que el pensum de Ingeniería Química, como todos los currículos de pregrado de la UPB, contempla cuatro ciclos de formación: básico de formación humanista (CBFH), propio a todo estudiante de la UPB; básico disciplinar (CBD), que incluye el estudio de las ciencias básicas; profesional (CP), que prepara para el desempeño laboral; y de integración $(\mathrm{Cl})$, que permite la conexión con los posgrados. Los ciclos están conectados de algunas maneras, por ejemplo, mediante los pre y correquisitos. La herramienta que se presenta en este trabajo hace parte de una metodología para observar que tan restrictivo es un pensum determinado, a partir de esto se pueden realizar modificaciones teniendo en cuenta los contenidos, habilidades y competencias de las actividades curriculares, su interconexión, y una equilibrada exigencia de los requisitos que dé lugar a un aumento de la flexibilidad en el plan de estudios.

La mayoría de los planes de estudio en Ingeniería Química presentan una malla curricular que emplea pre y correquisitos como metodología de control del avance curricular (University of Washington, 2015). Sin embargo, no se ha encontrado en la literatura revisada una estrategia de cuantificación que permita ponderar que tan restrictivo o que tan controlado pueda ser dicho plan de estudios. De acuerdo con lo mencionado, se sugiere que, antes de establecer un prerrequisito en el plan de estudios, se analice cuán necesario es y cómo afecta a los alumnos en el avance curricular. Es pertinente dimensionar los costos que esto implica para las instituciones educativas, la sociedad y el Estado, en consecuencia de la repitencia y el rezago en la titulación de los estudiantes (Pino Vera \& Soto Narbona, 2013).

\section{METODOLOGÍA}

Con miras a actualizar el conjunto de pre y correquisitos del plan de estudios vigente del Programa de Ingeniería Química de la UPB, y favorecer su flexibilidad, se elaboró un sistema de ponderación de restricciones con base en la severidad y el impacto en el flujo curricular que éstas representan. Para esto, se asignó un valor proporcional a los diferentes pre y correquisitos, ya sean de contenidos, formativos o reguladores.

En la Tabla 1 se presentan los pesos de restricción asignados a cada uno de los pre y correquisitos del plan de estudios. A mayor valor numérico, mayor es la restricción. Conviene recordar que en los pregrados de la UPB las actividades curriculares se califican en la escala 0.0-5.0, y se consideran aprobadas si la calificación definitiva es mayor o igual a 3.0. En el requisito formativo se da un peso de 1.0 para un curso con nota $\geq 2.0$ y un peso de 1.5 para un curso aprobado. Respecto al requisito de Ciclo Básico Disciplinar, el peso corresponde a la restricción neta del requisito de aprobar todos los cursos del CBD (ciencias básicas).

Tabla 1: Listado de requisitos y sus pesos de restricción

\begin{tabular}{|l|c|}
\hline \multicolumn{1}{|c|}{ Tipo de requisito } & Peso de restricción \\
\hline Correquisito por contenidos & 2.0 \\
\hline Prerrequisito por contenidos (Curso aprobado) & 1.5 \\
\hline Prerrequisito por contenidos (Curso con nota $\geq 2.0)$ & 1.0 \\
\hline Requisito regulador & 2.0 \\
\hline Requisito formativo & 1.001 .5 \\
\hline Requisito de Ciclo Básico Disciplinar & 4 \\
\hline
\end{tabular}

Sumando los pesos designados por esta métrica puede evaluarse tanto el nivel de restricción total de una sola actividad académica, como el de un conjunto de ellas (por ejemplo, el conjunto de las que componen lo que se llama en Colombia un semestre académico, normalmente compuesto por 16 a 18 créditos que se cursan simultáneamente en un período, bien sea enero-mayo o julio-noviembre). También se puede evaluar el nivel total de restricción de un conjunto completo de pre y correquisitos de un plan de estudios. Lo más 
interesante es que este sistema permite comparar el nivel de restricción de diferentes conjuntos de pre y correquisitos para un mismo plan de estudios (o incluso para diferentes planes de estudio). Para esto, se definió un indicador de comparación, llamado índice de restricción, que presenta el nivel total de las restricciones del plan de estudios con respecto al número total de créditos que lo componen (ecuación 1).

Índice de restricción $=\frac{\text { Nivel total de restricción }}{\text { Créditos totales }}$

\section{RESULTADOS Y DISCUSIÓN}

A partir de la métrica definida, se determinó el total de restricciones para cada uno de los 11 semestres que componen el actual plan de estudios de Ingeniería Química. Es de anotar que solo se tuvieron en cuenta los pre y correquisitos de los cursos que administra y que puede manipular directamente la Facultad, que son básicamente los del Ciclo Profesional. En la Figura 1 se presenta el resultado por semestre para el conjunto de pre y correquisitos vigente, y para una propuesta de actualización de dicho conjunto.

La concentración de la mayoría de restricciones entre el quinto y el noveno semestre se debe a que allí se ubican la mayoría de los cursos del ciclo profesional. El quinto semestre presenta el mayor valor de restricción para los requisitos vigentes, en contraste con la nueva propuesta de requisitos, donde el mayor valor de restricción se presenta en noveno semestre, a causa de cursos integradores del currículo, tales como Diseño de Reactores II y Diseño de Procesos I.

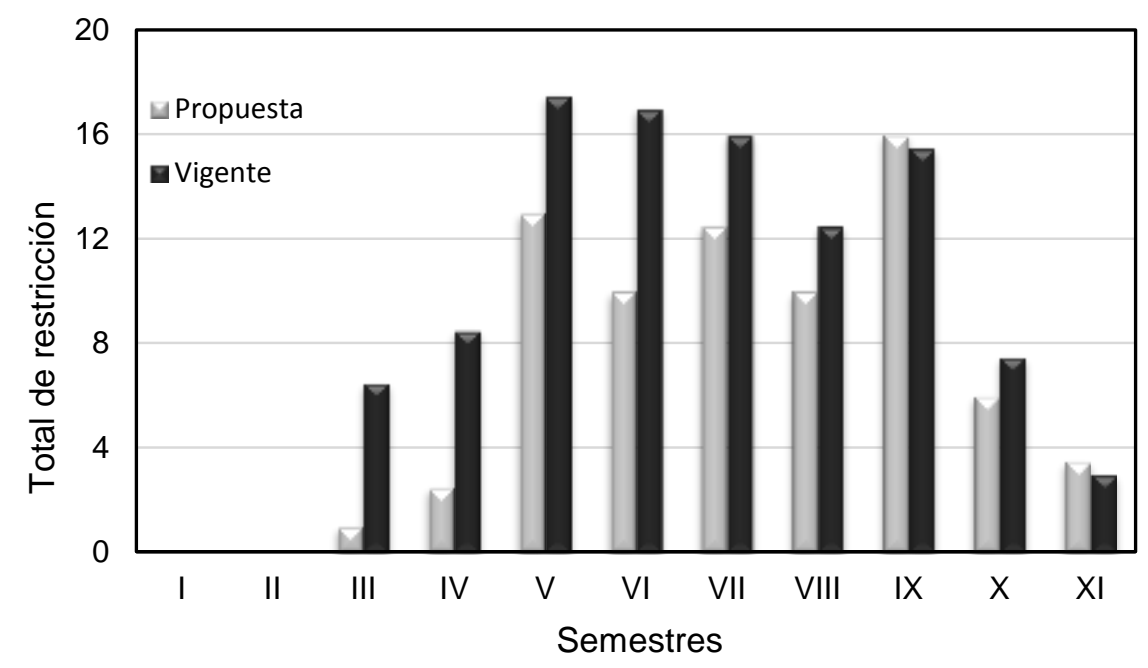

Fig. 1: Total de restricción por semestre para el conjunto de requisitos vigente del plan de estudios de Ingeniería Química de la UPB) y para la propuesta de la actualización de este conjunto

Como ejemplo detallado del cambio que se logra de un conjunto de pre y correquisitos a otro (del vigente a la propuesta, en este caso), en la Figura 2 se presenta una comparación entre los valores de restricción para los cursos del sexto semestre académico, semestre para el cual se encuentra la mayor disminución de restricciones desde el punto de vista de la propuesta. El motivo es que se cambia el requerimiento de haber aprobado todos los cursos del CBD por la aprobación de algunos cursos específicos, obteniéndose mayor flexibilidad.

El único curso del sexto semestre que, con la nueva propuesta de requisitos, aumenta de valor de restricción, es transferencia de calor. Esto se debe a que fue seleccionado como punto de control que articula los ciclos CP y CBD que tiene como prerrequisitos formativos la aprobación de ecuaciones diferenciales y cálculo vectorial.

Al calcular el Índice de restricción total para el conjunto de pre y correquisitos vigente para el plan de estudios de Ingeniería Química, y el de la propuesta actualizada para este mismo conjunto, se observa que se obtiene una reducción del $30 \%$ con la nueva propuesta: el Índice pasa de 0.54 a 0.38 , tal como se presenta en la Figura 3. 


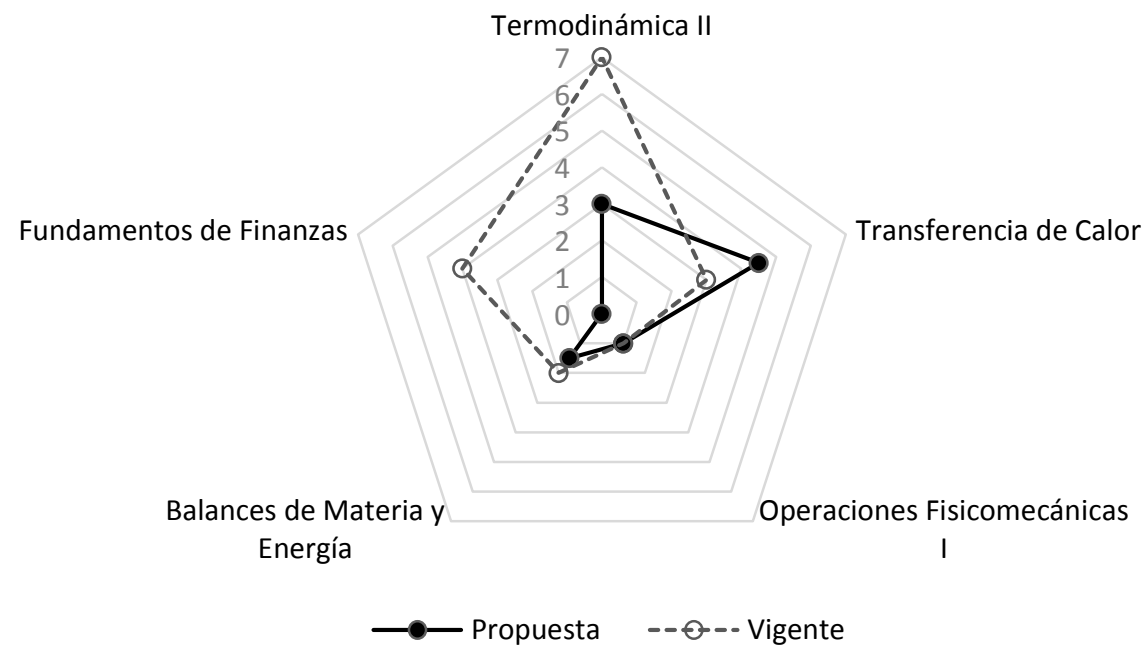

Fig. 2: Comparación del valor de restricción para los cursos de sexto semestre según la propuesta para el conjunto de pre y correquisitos y según el conjunto de requisitos vigente.

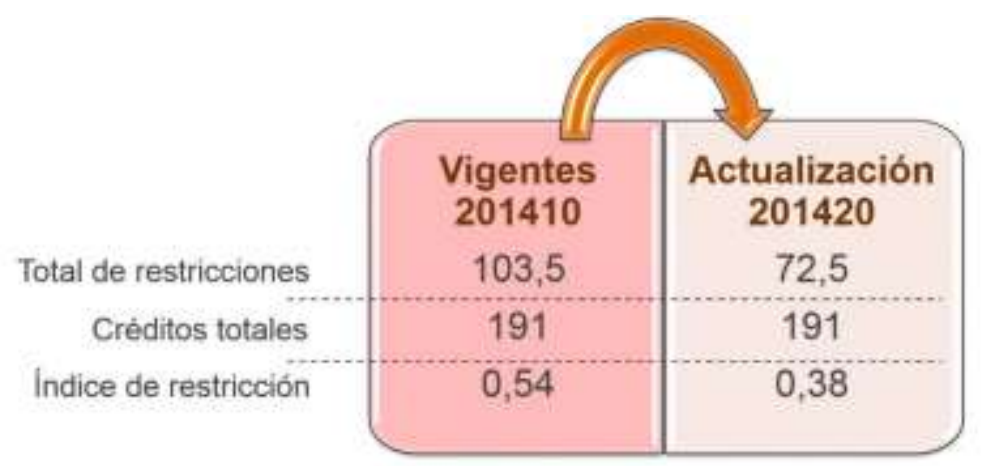

\section{Reducción del $30 \%$ en las restricciones del Plan de estudios}

Fig. 3. Restricciones totales e índice de restricción para el conjunto de requisitos vigentes del plan de estudios de ingeniería química y la propuesta de actualización de este conjunto.

El Consejo de la Facultad de Ingeniería Química aprobó entonces la actualización del conjunto de requisitos para el plan de estudios. Con esta disminución de restricciones se permite al estudiante una mayor flexibilidad para la selección del orden en el que puede tomar cursos, disminuyendo las solicitudes especiales sobre la omisión de un requisito curricular determinado, y la cancelación automática indeseada de correquisitos cuando el estudiante decide cancelar un curso. En última instancia, se espera que esto redunde también en una disminución de la deserción académica.

\section{CONCLUSIONES}

La valoración del nivel de restricción de los requisitos de las actividades académicas de un plan de estudios permite un mejor conocimiento de su influencia en el flujo curricular, lo que posibilita tomar decisiones en pro de un currículo más flexible, con miras a elevar los niveles de permanencia y de egreso de los estudiantes en las carreras universitarias. Todo esto cuidando que no se afecte el nivel de calidad del egresado.

La metodología propuesta puede ser implementada en diferentes programas académicos para realizar comparaciones de la severidad de las restricciones de diferentes conjuntos de requisitos para un mismo plan de estudios, o de los conjuntos de requisitos para las diferentes versiones del pensum. 


\section{REFERENCIAS}

Abou-Sayf, F. K., Does the Elimination of Prerequisites Affect Enrollment and Success?, doi: 10.1177/0091552108319539, Community College Review 36(1), 47-62 (2008)

Baard, V. y Watts, T., The value of prerequisites: $A$ link between understanding and progression, http://www.ejbest.org/upload/Baard,_Vicki.pdf; ISSN: 1835-9132, e-Journal of Busines Education \& Scholarship of teaching, 2(1), 1-10 (2008)

Castrillón, F. y B. Garcés, Camino y logros de una transformación curricular en Ingeniería Química, World Engineering Education Forum - WEEF 2012, 257, Buenos Aires, Argentina, 15 a 18 de octubre (2012)

California Office of Administrative Law, California Code of Regulations. Program, course and class classification and standards (5 CCR § 55002), http://www.calregs.com/linkedslice/default.asp?SP=CCR1000\&Action=Welcome, Acceso: 10 de marzo (2008)

Duque, M., J. Celis y A. Camacho, Cómo lograr la alta calidad en la educación de los ingenieros: una visión sistémica, Revista Educación en Ingeniería, Diciembre 2011 (12), 48-60, (2011)

Pino Vera, T. y M. F. Soto Narbona, El prerrequisito en el avance curricular: Estudio de caso tras movilizaciones estudiantiles del 2011 en Chile,

http://revistas.uis.edu.co/index.php/revistadocencia/article/view/4222, ISSN: 2145-8537, Docencia

Universitaria 14, 29-42 (2013)

Ruíz, J. M., G. Barreto y R. Blanco, Flexibilidad en planes de estudios universitarios, Pedagogía y Saberes, 26, 9-17, (2007)

Soria, K. M. y L. Mumpower, Critical Building Blocks: Mandatory Prerequisite Registration Systems and Student Success, NACADA Journal, 32(1), 30-42, (2012)

Universidad de Antioquia, Oferta de programas de pregrado,

http://portal.udea.edu.co/wps/portal/udea/web/inicio/estudiar-udea/pregrado/oferta, Medellín, Acceso: 17 de julio de 2015

Universidad de la Sabana (2015),

http://www.unisabana.edu.co/fileadmin/Documentos/Ingenieria_Quimica/Mallas_WEB_2015_con_core_curr iculum_IQ.pdf, Bogotá D.C, Acceso: 17 de julio de 2015.

Universidade de Sao Paulo, Pre-Requisitos 2011 Noturno - Unifesp,

https://www.yumpu.com/pt/document/view/27576114/prac-requisitos-2011-noturno-unifesp, Acceso: 10 de Abril (2015)

Universidad Pontificia Bolivariana, Modelo pedagógico integrado (2009), http://www.upb.edu.co/pls/portal/docs/PAGE/GP_REPOSITORIO_IMAGENES/PG_RIMG_MEDELLIN/MOD ELO\%2OPEDAGOGICO.PDF, Medellín, Acceso: 15 de junio $201 \overline{4}$.

University of Washington, Chemical Engineering Graduation Requirements, www.cheme.washington.edu, Washington, Acceso: 1 de febrero (2015)

Woosley, S. A. y T. Callahan, The effects of prerequisites and curricular continuity: domestic versus imported, College Student Journal, 45(3), 534-535, (2011)

Zambrano, J., R. Velásquez y F. Sáenz, Tendencias de la Educación en Ingeniería, AVANCES Investigación en Ingeniería, 2, 4-16, (2005) 
